\title{
Environmental aspects of using nanomaterials
}

\begin{abstract}
The paper presents the nanotechnology as a one of main fields of modern science. Since nanotechnology accompanies the everyday life, one should focus on its benefits and disadvantages. Some nanoparticles exhibit animicrobial properties and using them definitely improves the quality of life. Silver nanoparticles are used in many consumer products which serve as antibacterial or antifungal agents. However, due to the fact that the market of nanomaterials is constantly growing, most of living organisms may be exposed on nanoparticles. Silver nanoparticles may be easily accumulated in various organs, such as liver, kidneys, lungs and others. Despite the fact that their activity may not be seen immediately, their impact on living matter may have negative consequences in the future. Hazard and responses issues are raised in the review.
\end{abstract}

Volume 4 Issue 5 - 2017

\author{
Marcin Banach, Jolanta Pulit Prociak \\ Faculty of Chemical Engineering and Technology, Cracow \\ University of Technology, Poland
}

\begin{abstract}
Correspondence: Marcin Banach, Institute of Chemistry and Inorganic Technology, Cracow University of Technology, Warszawska 24, Cracow, Poland, Tel 48I2628286I, Fax
\end{abstract} 48126282035, Email marcinbanach@chemia.pk.edu.pl

Received: February 23, 2017 | Published: May 08, 2017

Keywords: nanotechnology, nanosilver, antimicrobial properties, toxicity

\section{Introduction}

Nanotechnology is a rapidly growing field of science, which is particularly popular. ${ }^{1}$ Taking into consideration the use of nanotechnology, it can be concluded that we have contact with it in the most part of everyday life. Nanocomponents play a significant role in various fields of science, which in consequence is reflected in the improved quality of human life.

According to the statement issued by the European Commission, the global amount of manufactured nanomaterials is close to 11.5 million tons, which is equivalent to their market value reaching 20 billion Euro per year (this estimate also apply to soot and amorphous silica). It is estimated that the current global market for nanomaterials is equal to 300000 tons up to 1.6 million tons. ${ }^{2}$ The largest market share is in the Asian region (approx. 34\%), North American (approx. $31 \%$ ) and Europe (approx. 30\%). Global demand for nanomaterials increases from year to year. This entails an inevitable passage. ${ }^{3}$ There is no doubt that the presence of nanoparticles in many consumer products generally improves the quality of life, providing numerous benefits and economic utility. The nanoparticles have been introduced in the formulation of hundreds of different types of products.

\section{Discussion}

Taking into consideration the nano-sized particle size, one may not observe their chemical and physical effects on living matter. ${ }^{4}$ Nanomatter penetration into the environment and the specific activity can cause degradation effects. ${ }^{5}$ Accordingly, there is a need of broader studies on the characteristics of the nanoparticles, both in terms of their physicochemical properties and biological activity. ${ }^{6}$ It is also necessary to modify the processes for obtaining them, in order to improve the control of these properties or develop such formulas that will not have the harmful activity on living matter or will be limited while maintaining the functionality of materials. The impact of nanoparticles on the environment may take various forms. It is worth noting that weather factors such as temperature or humidity can also affect the physical properties of nanoparticles (size and stability). ${ }^{7}$ These processes are long lasting, but one should be aware that once nanoparticles are introduced into the environment it may result in the contamination of soil, surface water and groundwater as well as flora and fauna. Living organisms may be not exposed to nanomaterials during their life, but toxicological concerns can be a problem in the future, the process of penetration of nanomaterials reach the stage of direct contact with successive generations of living matter ${ }^{1}$ Understanding the life cycle of nanoparticles in the environment and their chemical stability is an important step in the process of determining the effects of nanomaterr on living organisms. ${ }^{8}$ Despite the lack of thorough research of threat from nanomaterials one can determine the course of research framework, which assumes:

a. Physicochemical characteristics of particles which may have a negative impact on living matter,

b. Familiarize themselves with the life cycle of nanomaterials and the way their penetrate inside the organism,

c. Selection of appropriate techniques for measuring the degree of organisms exposure to the nanomaterials,

d. Defining the rules of deploying nanoparticles in different parts of living organisms,

\section{e. Developing mechanisms causing medical conditions. ${ }^{1}$}

In the literature two main hypotheses explaining the toxic effects of nanoparticles on living organisms can be found. The first hypothesis is that the harmful activity of nanoparticles is associated with the release of ions from these particles..$^{9,10}$ The second says that cause of the toxicity of nanomaterials is associated with the formation of ROS (reactive oxygen species). The free-radicals are able to damage any components of the cell, and initiate the production of more and more kinds of reactive oxygen species. ${ }^{11}$ The resulting free radicals are able to oxidize the double bonds of fatty acids in cell membranes, resulting in an increased permeability of the membrane, which contributes to the osmotic stress. ${ }^{12}$ ROS can inhibit the enzyme activity by binding to them, change the DNA helix, which can lead to cell death. Preparation of more reactive forms of oxygen is associated with the high surface area of nanoparticles as compared to their bigger analogues. ${ }^{12}$ Nanomaterials may also cause damage to cell membranes, may oxidize proteins, have genotoxic effect as well as interfere the energy transfer. ${ }^{11}$

Metallic nanoparticles, due to their unique properties, are seen as a leader in the fight against pathogenic microbial action. Nanometals are characterized by the strong effects of slowing down their functioning. They are used in a wide range of scientific fields such as biotechnology, medicine, pharmacy, ecology, electronics and others. Metallic nanoparticles are also used in the agriculture, veterinary medicine, food industry and cosmetology. ${ }^{13}$ 
Table 1 presents the list of microorganisms to which the biocidal activity of nanosilver has been confirmed. ${ }^{14-23}$ Silver nanoparticles are effective factor for destroying a wide range of Gram-negative and Gram-positive bacteria. It also has bactericidal activity against strains resistant to antibiotics. ${ }^{24}$ Recent studies have shown that the use of silver nanoparticles in combination with certain antibiotics, such as penicillin G, amoxicillin, erythromycin, clindamycin and vancomycin, creates synergies against Escherichia coli or Staphylococcus aureus. ${ }^{25}$ Scientific research has shown that silver nanoparticles can be an effective weapon in the fight against viruses, ${ }^{26}$ as it inhibits its replication. The efficiency of the processes leading to destruction of viruses strictly depends on the shape and size of nanoparticles. ${ }^{27}$

Table I Microorganisms to which the biocidal activity of nanosilver has been confirmed

\begin{tabular}{lll}
\hline Bacteria & Viruses & Fungi \\
\hline Acinetobacter $^{14}$ & HIV-I I & Aspergillus $^{22}$ \\
Escherichia $^{15}$ & Influenza virus $^{21}$ & Candida $^{23}$ \\
Pseudomonas $^{14}$ & & Saccharomyces $^{24}$ \\
Salmonella $^{16}$ & \\
Bacillus $^{16}$ & \\
Enterococcus $^{17}$ & \\
Listeria $^{18}$ & \\
Staphylococcus & \\
Streptococcus & \\
\end{tabular}

The European Commission on Nanomaterials claims that there is a huge need of monitoring the quality and quantity of nanoparticles that are introduced to consumer products. A regulation was issued that all types of nanoparticles that are used in cosmetic products as UV-filters, colourants and preservatives must be registered. ${ }^{28}$ It is due to the fact that metallic nanoparticles can penetrate into the body through the skin, respiratory and digestive systems. As previous studies disclosed, particularly silver nanoparticles tend to accumulate in different organs, especially the liver, kidneys, lungs and others. The presence of nanoparticles in the liver may be particularly danger. This organ is unable to expel the silver nanoparticles, which can lead to release into the other organs. Reported accumulation of nanoparticles in the lungs can also have negative effects in the future. Undoubtedly, it is necessary to conduct further research into the toxicity of metallic nanoparticles on living organisms. Full scientific information is not yet available, since the developed applications are incomplete. It is necessary to implement the full test cycle, on the basis of which it will be possible to determine the actual amount of metallic nanoparticles permeating into the environment. In the next phase it is necessary to perform tests to determine the degree of nanometals accumulation in living matter. It should also broaden knowledge of their impact on living organisms. Implementation of the full test cycle will be the basis for further actions aimed primarily at protecting the environment. If the alarming data will be confirmed, it is also necessary to provide a method for environment renaturisation and protection against a possible threat from nanomaterials accumulated in the future. In order to recognize the emerging threat posed by metallic nanoparticles one should take into account four factors: hazard identification, assessment of the degree of toxicity, exposure assessment and hazard characteristics. The nanometric materials are varied in size, elemental composition, particle size distribution, crystalline structure, surface area, stability and capacity of interaction of the complexing solutions such as blood or bacterial solution.

\section{Conclusion}

Considering the above, it is concluded that it is also important to understand the associated toxicological properties. The small size of nanomaterials will introduce new properties that can be both beneficial and environmentally harmful. A more complete description of how these parameters affect the biological activity of nanoparticles is necessary. Balancing the risks and benefits of nanomaterials is essential to safe and study their development. There is a general belief that the study of the toxicity of nanomaterials is falling behind their desire for commercial use. There is an urgent need to standardize the evaluation of the safety of nanomaterials, which would facilitate the assessment of exposure to nanomaterials and the risks arising from their use. The purpose is to secure the assimilation of nanotechnology in the wider society. Information obtained during the study will enable the systematization of knowledge and targeted development, including obtaining products of defined quality, intentional, and their proper use, control over the introduction of nanoparticles in the life cycle and their controlled presence in the environment.

\section{Acknowledgements}

None.

\section{Funding}

None.

\section{References}

1. Lu K. Nanoparticulate materials. Synthesis, characterization, and processing. (1st edn), John Willey \& Sons, New Jersey, USA. 2013;123.

2. Porter A, Youtie J. How interdisciplinary is nanotechnology? J Nanopart Res. 2009;11(5):1023-1041.

3. http://eur-1ex.europa.eu/legal-content/EN/TXT/ $\mathrm{PDF} /$ ?uri $=$ CELEX:52012DC0572\&from $=\mathrm{PL}$

4. Remédios C, Rosário F, Bastos V. Environmental nanoparticles interactions with plants: morphological, physiological, and genotoxic aspects. Journal of Botany. 2012;p. 8.

5. Ray PC, Yu H, Fu PP. Toxicity and environmental risks of nanomaterials: Challenges and future needs. J Environ Sci Health C Environ Carcinog Ecotoxicol Rev. 2009;27(1):1-35.

6. Stankic S, Suman S, Haque F, et al. Pure and multi metal oxide nanoparticles: synthesis, antibacterial and cytotoxic properties. $J$ Nanobiotechnol. 2016;14(1):73-93.

7. Huang Y, Lenaghan SC, Xia L, et al. Characterization of physicochemical properties of ivy nanoparticles for cosmetic application. $J$ Nanobiotechnol. 2013;11:3.

8. González GD, Janer G, Vilar G, et al. The life cycle of engineered nanoparticles. Advances in Experimental Medicine and Biology. 2017;947:41-69.

9. Li M, Zhu L, Lin D. Toxicity of ZnO nanoparticles to Escherichia coli: mechanism and the influence of medium components. Environ Sci Technol. 2011;45(5):1977-1983.

10. Sawinska Z, Khachatryan K, Sobiech L, etal. Use of silver nanoparticles as a fungicide. Przem Chem. 2014;93(8):1472-1474.

11. Klaine SJ, Alvarez PJJ, Batley GE, et al. Nanomaterials in the environment: Behavior, fate, bioavailability, and effects. Environ Toxicol Chem. 2008;27(9):1825-1851.

12. Bhatt I, Tripath BN. Interaction of engineered nanoparticles with various components of the environment and possible strategies for their risk assessment. Chemosphere. 2011;82(3):308-317.

13. Jie J, Zhang W, Bello I, et al. One-dimensional II-VI nanostructures: synthesis, properties and optoelectronic applications. Nano Today. 2010;5(4):313-336 
14. Niakan S, Niakan M, Hesaraki S, et al. Comparison of the Antibacterial effects of nanosilver with 18 antibiotics on multidrug resistance clinical isolates of Acinetobacter baumannii. Jundishapur J Microbiol. 2013;6(5): e8341.

15. Li WR, Sun Q, Zhan J, et al. Soil-covered strategy for ecological restoration alters the bacterial community structure and predictive energy metabolic functions in mine tailings profiles. Applied Microbiology and Biotechnology. 2010;101(6):2549-2561.

16. Shahrokh S, Emtiazi G. Toxicity and unusual biological behavior of nanosilver on Gram-positive and negative bacteria assayed by Microtiter-Plate. Eur J Biol Sci. 2011;1(3):28-31.

17. Lotfi M, Vosoughhosseini S, Ranjkesh B, et al. Antimicrobial efficacy of nanosilver, sodium hypochlorite and chlorhexidine gluconate against Enterococcus faecalis. African Journal of Biotechnology. 2011;10(35): 6799-6803.

18. Zarei M, Jamnejad A, Khajehali E. Antibacterial effect of silver nanoparticles against four foodborne pathogens. Jundishapur $J$ Microbiol. 2014;7(1): e8720.

19. Ahangaran MG, Firouzabadi MSS, Firouzabadi MS, et al. Evaluation of antiseptic role of one nanosilver based drug as a new therapeutic method for treatment of Bumblefoot in Pheasant (Phasianus colchicus). Glob Vet. 2012;8(1):73-75.

20. Cheng L, Zhang K, Weir MD, et al. Effects of antibacterial primers with quaternary ammonium and nano-silver on Streptococcus mutans impregnated in human dentin blocks. Dent Mater. 2013;29(4):462-472.
21. Mehrbod P, Motamed N, Tabatabaian M, Soleimani Estyar R, Amini E, et al. (2009) In vitro antiviral effect of Nanosilver on influenza virus. Daru. 2009; 17(2):88-93.

22. Nagash N, Safari M, Hajmehrabi P. Comparison of nanosilver inhibitory effects growth between Aspergillus niger and E. coli. Indian $\mathrm{J} \mathrm{Sci}$ Technol. 2012;5(3):2448-2450.

23. Keuk KJ, Sung WS, Moon SK, et al. Antifungal effect of silver nanoparticles on dermatophytes. $J$ Microbiol Biotechnol. 2008;18(8):1482-1484.

24. Wright JB, Lam K, Hansen D, et al. Efficacy of topical silver against fungal burn wound pathogens. Am J Infect Control. 1999;27(4): 344 350

25. Shahverdi AR, Fakhimi A, Shahverdi HR, et al. Synthesis and effect of silver nanoparticles on the antibacterial activity of different antibiotics against Staphylococcus aureus and Escherichia coli. Nanomedicine. 2007;3(2):168-171.

26. Wijnhoven SWP, Peijnenburg WJGM, Herberts CA, et al. Nanosilver - a review of available data and knowledge gaps in human and environmental risk assessment. Nanotoxicology. 2009;3(2):109-138.

27. Elechiguerra JL, Burt JL, Morones JR, et al. Interaction of silver nanoparticles with HIV-1. J Nanobiotechnol 6. 2005.

28. http://ec.europa.eu/growth/sectors/cosmetics/products/nanomaterials 